\title{
NOTE ON A THEOREM OF DICKSON
}

\author{
HAROLD N. SHAPIRO
}

Though the problems concerning perfect and multiply perfect numbers are among the oldest of number theory, very little progress has been made in the investigation of these numbers. A $k$-ply perfect number is one for which $\sigma(n)=k n$ where $\sigma(n)$ is the sum of the divisors of $n$. The case $k=2$ is that of the perfect numbers. Though the form of the even perfect numbers is completely determined $[1],{ }^{1}$ the question of whether or not there exists any odd perfect numbers is still a complete mystery. Sylvester [2] has shown that an odd perfect number must have at least five distinct prime factors, and Dickson [3] has proved that there are at most a finite number of odd perfect numbers having any given number of distinct prime factors. More generally, defining "primitive non-deficient" numbers to be those integers $n$ for which

$$
\sigma(n) / n \geqq 2
$$

and such that (1) does not hold for any proper divisor of $n$, Dickson showed that there are at most a finite number of odd primitive nondeficient numbers having a given number of distinct prime factors. In this note we shall give a simpler proof of Dickson's theorem; in fact prove a more general theorem which includes Dickson's as a special case.

Definition 1. An integer $n$ shall be called $k$-non-deficient ( $k$ any positive real number) if

$$
\sigma(n) / n \geqq k
$$

and $k$-deficient otherwise.

DEFINITION 2. An integer $n$ shall be called primitive $k$-non-deficient if $n$ is $k$-non-deficient, and all proper divisors of $n$ are $k$-deficient.

Our generalization of Dickson's theorem may then be stated as:

THEOREM 1. There are at most a finite number of primitive k-nondeficient numbers $n$ such that

(a) if $k$ is rational, $n$ is relatively prime to the numerator of $k$; and

(b) the number of distinct prime factors of $n$ is fixed.

Proof. We assume that there are an infinite number of such primi-

Received by the editors April 16, 1948.

1 Numbers in brackets refer to the bibliography at the end of the paper. 
tive $k$-non-deficient numbers which have $s$ distinct prime factors. Writing each of these in the form

$$
n=p_{1}^{\alpha_{1}} \cdots p_{s}^{\alpha_{s}}, \quad p_{1}^{\alpha_{1}}<p_{2}^{\alpha_{2}}<\cdots<p_{s}^{\alpha_{s}},
$$

we note that at least $p_{s}^{\alpha_{s}} \rightarrow \infty$ over this sequence. Hence from these $n$ can extract an infinite subsequence $n_{j}$ such that

$$
n_{j}=p_{1}^{\alpha_{1}} \cdots p_{r}^{\alpha_{r}} p_{r+1}^{\alpha_{r+1}, j} \cdots p_{t}^{\alpha_{t j}} p_{t+1, j}^{\alpha_{t+1, j}} \cdots p_{s j}^{\alpha_{s j}}
$$

where

(I) $p_{i}^{\alpha_{i}}$ are fixed independently of $j$ for $i=1, \cdots, r$;

(II) $\dot{p}_{i}$ are fixed independently of $j$, and

$$
\lim _{j \rightarrow \infty} \alpha_{i j}=\infty
$$

for $i=r+1, \cdots, t$; and

$$
\lim _{j \rightarrow \infty} p_{i j}=\infty
$$

for $i=t+1, \cdots, s$.

It is to be noted that any one of these sets may actually be empty, and all that we know a priori is that at least one of the two groups (II), (III) is not empty.

Now first we shall show that the group (III) cannot be empty. For if it were, the primes dividing each $n_{j}$ are the same as those dividing $n_{0}$. Thus by taking $j$ sufficiently large we obtain $\alpha_{i j} \geqq \alpha_{i 0}, i=1, \cdots, s$, where the strict inequality holds for at least one $i$; so that $n_{0}$ is a proper divisor of $n_{j}$. But this contradicts the assumption that both $n_{0}$ and $n_{j}$ are primitive $k$-non-deficient.

Since (III) is non-empty, and the $n_{j}$ primitive $k$-non-deficient we have

$$
\prod_{\text {(I) }} \prod_{\text {(II) }} \prod_{\text {(III) }} \frac{p_{i j}^{\alpha_{i j}+1}-1}{\left(p_{i j}-1\right) p_{i j}^{\alpha_{i j}}} \geqq k>\prod_{\text {(I) }} \prod_{\text {(II) }} \prod_{i=t+1}^{s-1} \frac{p_{i j}^{\alpha_{i j}+1}-1}{\left(p_{i j}-1\right) p_{i j}^{\alpha_{i j}}} .
$$

Then taking the limit as $j \rightarrow \infty$, we get

$$
\prod_{\text {(I) }} \frac{p_{i}^{\alpha_{i}+1}-1}{\left(p_{i}-1\right) p_{i}^{\alpha_{i}}} \prod_{\text {(II) }} \frac{p_{i}}{p_{i}-1} \geqq k \geqq \prod_{\text {(I) }} \frac{p_{i}^{\alpha_{i}+1}-1}{\left(p_{i}-1\right) p_{i}^{\alpha_{i}}} \prod_{\text {(II) }} \frac{p_{i}}{p_{i}-1}
$$

which in turn yields

$$
\prod_{\text {(I) }} \frac{p_{i}^{\alpha_{i}+1}-1}{p_{i}-1} \prod_{\text {(II) }} p_{i}=k \prod_{\text {(I) }} p_{i}^{\alpha_{i}} \prod_{\text {(II) }}\left(p_{i}-1\right) .
$$


If $k$ is irrational, this gives an immediate contradiction. If $k$ is rational and group (II) is not empty, the fact that $\prod_{\text {(II) }} p_{i}$ is relatively prime to the numerator of $k$ implies that $\Pi_{\text {(II) }} p_{i}$ divides $\prod_{\text {(II) }}\left(p_{i}-1\right)$. This is clearly impossible. On the other hand, if (II) is empty (3) becomes

$$
\prod_{(\mathrm{I})} \frac{p_{i}^{\alpha_{i}+1}-1}{p_{i}-1}=k \prod_{(\mathrm{I})} p_{i}^{\alpha_{i}} .
$$

But this in turn is also impossible since $\Pi_{\text {(I) }} p_{i}^{\alpha_{i}}$ is a proper divisor of $n_{0}$ and must be $k$-deficient. This contradiction completes the proof of the theorem.

By an entirely analogous argument the following generalization of another theorem of Dickson [4] may be obtained.

THEOREM 2. For $k$ rational, there are at most a finite number of primitive $k$-non-deficient integers $n$ such that

(a) $p \mid$ (numerator of $k$ ), and $p^{\alpha} \| n$ implies $\alpha<C$, a fixed constant; and

(b) the number of distinct prime factors of $n$ is fixed.

\section{BIBLIOGRAPHY}

1. E. Landau, Vorlesungen über Zahlentheorie, vol. I, p. 19.

2. J. J. Sylvester, Sur l'impossibilité de l'existence d'un nombre parfait impair qui ne contient pas au moins 5 diviseurs premiers distincts, C. R. Acad. Sci. Paris vol. 106 (1888) pp. 522-526.

3. L. E. Dickson, Finiteness of the odd perfect and primitive abundant numbers with $n$ distinct prime factors, Amer. J. Math. vol. 35 (1913) pp. 413-422.

4. - Even abundant numbers, Amer. J. Math. vol. 35 (1913) pp. 423-426.

Princeton University 